\title{
Knowledge of Mothers Regarding Child Abuse In Rural areas
}

\author{
Rajendra K \\ Assistant Professor, Department of Sociology, Government First Grade College, Manki, Uttar Kannada, INDIA,
}

\begin{abstract}
Approximately 702,000 children were confirmed by child protective services as being victims of abuse and neglect in 2014. At least one in four children have experienced child neglect or abuse (including physical, emotional, and sexual) at some point in their lives, and one in seven children experienced abuse or neglect in the last year. Very few studies have highlighted the turmoil burning issue of child abuse prevailing in rural areas and an attempt is made to determine the knowledge of rural mothers on child abuse.
\end{abstract}

Key words: child abuse, mother, rural area

\section{INTRODUCTION}

Growth and development are extremely important and unique aspects of childhood. Growth refers only to physical development where as development refers to the way in which children attain the ability to perform more complex action as they get older.Issues that children are facing during childhood are violence through indoctrinations, poverty life as refugees, lack of access to education, child neglect, child labour, child prostitution, internet child pornography, slavery, military use of children. Applying the label of child maltreatment implies a judgement about social deviances and brings to bear both personal and social values regarding parenting. Implicit in this process are judgement about circumstances harmful in children, minimal requirement for development and other aspects of the child that are important for the society to protect.

The broad term child maltreatment includes intentional physical abuse or neglect, emotional abuse and sexual abuse of children usually by adults.

Child abuse is one of the most significant social problems affecting children and parent. Child abuse may be the only type of violence in the family. Maltreatment triggered by the child misconduct, including locking up, cursing, belittling and beating was reported by a high proportion of mothers.

There were 702,000 victims of child abuse and neglect reported to child protective services (CPS) in 2014. The youngest children are the most vulnerable with about $27 \%$ of reported victims being under the age of three worldwide.In Indian culture, there is a high expectation for child's obedience and discipline and violating these rules provides a strong justification for punishment. This probably is the reason why a proportion of mothers fail to pursue such behaviours as abusive. Indeed the positive correlation between abusive behaviour and the belief is corrective punishment explain much about child maltreatment.

\section{OBJECTIVES}

1. Assess the level of knowledge of the mothers regarding child abuse.

2. Find the association between level of knowledge and demographic variables of the study.

\section{MATERIALS AND METHOD}

The source of primary data was collected from four villages in Udupi district. Convenience sampling technique was adopted in the selection of the samples. A descriptive survey approach was used in the study. The target population comprised of 100 mothers who gave birth to minimum one living child from rural areas. The respondents were interviewed using structured knowledge questionnaire. Knowledge is taken as the dependent variable and the independent variables considered are age, religion, occupation, education, income. The data was analysed using SPSS version 16.0.

\section{FINDINGS}

When considering the age group distribution, majority (31\%) of the mother's age ranged between 20-25 years, about fourteen percent of the mothers were above thirty six years of age. About half of the mothers had primary education and nearly three percent were illiterate. The respondents were predominantly Hindu (65\%). About 23\% were Muslims and remaining twelve percent Christians. The current occupational status among the mothers showed that fifty one percent of them were housewives and remaining twenty seven percent of them were still working. The mothers were belonging to nuclear family. Three fourth of the respondents family reported a monthly income ranging from Rs. 3500/- to Rs. 5000/- and one fourth had a monthly income of more 
than Rs. 5000/-. The important demographic attribute is the number of living children which revealed around $3.6 \%$ of the respondents have one single child and more than $56 \%$ of the respondents had two to three children and almost forty percent of them have four to five children. More than half of the respondent's children fathers were substance abusers.

Ninety six percent of the respondents were familiar with the term child abuse and majority have seen or heard about the matter in the television or read in the newspaper.

Approximately forty three percent of the respondents were able to identify correctly the types of child abuses. Only three percent of them correctly recognised the signs and symptoms of different type of abuses.

When the respondents were asked what will be their reaction if a child is abused in any form, a large proportion of them reported that they would immediately bring the matter to the notice of police and take the child to the hospital for further treatment.

An open ended question with regard to the risk factors revealed significant findings such as social support, maternal psychological adjustment, maternal preparation for parenting, parental marital status, parental education level, family rearing patterns and family atmosphere.

The chi square test of association showed no significant association between child abuse and age, education, occupation and religion, but a significant association was found between type of family and child abuse.

\section{CONCLUSIONS}

This study aimed at assessing the knowledge of rural mothers in terms of types, recognition and referral of abuses. Every country in the world has its own law with regard to reporting of child abuse. An awareness with regard to the reporting of the child abuse can increase the frequency and identify the lack of knowledge public has and over shadow the stigma it is attached with. To combat this issue it is essential that multi approach programs in the form of improved parenting skills and safe home environment is being created.

\section{REFERENCES}

[1] Chiocca, E (1998), “Child abuse and neglect: A status Report”, Journal of Paediatric Nursing, 13(2):128130.

[2] ACOG educational bulletin (2001), "Adult manifestation of childhood abuse", International Journal of Gynaecology and Obstetrics, 74:311-320.

[3] HALL,L.A and Sarchs B (1998), "Mothers potential for child abuse, the roles of child abuse and social resources", Nursing Research,47(2)

[4] Finkelhor D, Turner, H.A, Shattuck A, Hamby, S.L (2015), "Prevalence of childhood exposure to violence, crime, and abuse: Results from the National Survey of Children's Exposure to Violence", JAMA Paediatric, 169(8): 746-754. 